\title{
Epstein-Barr Virus-associated Mixed Lymphoepithelioma-like Carcinoma and Adenocarcinoma of the Gall Bladder: An Unusual Entity
}

\author{
Niharika Jain, Surbhi Goyal, Puja Sakhuja and Sundeep Saluja ${ }^{1}$ \\ Departments of Pathology and Gastrointestinal Surgery ${ }^{1}$, Govind Ballabh Pant Institute of Postgraduate Medical Education and Research, \\ New Delhi, India
}

\begin{abstract}
Schmincke described lymphoepithelioma as an undifferentiated carcinoma with abundant lymphoid stroma in the nasopharynx. Tumors with a similar histomorphology in extrapharyngeal areas have been referred to as lymphoepithelioma-like carcinoma (LELC). The association of an Ebstein-Barr virus (EBV) infection with lymphoepithelioma is well established in the nasopharynx but not so well at the extrapharyngeal sites. Only four cases of LELC have been reported in the gall bladder, of which all were negative for the EBV. This paper reports the first case of an EBV-associated mixed gall bladder carcinoma exhibiting a distinct phenotype of LELC and adenocarcinoma with mucinous differentiation. The EBV was confirmed by the strong granular membranous and cytoplasmic expression of LMP-1 (latent membrane protein-1) on immunohistochemistry and nuclear EBER RNA on chromogen in-situ hybridization in the tumor. This is the first case of LELC positive for EBV in the gall bladder. LELC has a more favorable prognosis than conventional adenocarcinoma or squamous cell carcinoma, irrespective of the site. Although a higher T stage and nodal metastasis were exceptional in the present case in contrast to the previous cases, the EBV-associated lymphocytic response might limit the disease spread and confer better overall survival and prognosis in these patients. Nevertheless, more prospective studies with a larger cohort will be needed to understand the pathogenesis, biological behavior, and prognosis of this rare entity. (Korean J Gastroenterol 2021;78:188-194)
\end{abstract}

Key Words: Gallbladder neoplasm; Lymphoepithelioma-like carcinoma; Epstein-Barr virus; Adenocarcinoma

\section{INTRODUCTION}

Schmincke $^{1}$ described lymphoepithelioma as an undifferentiated carcinoma with abundant lymphoid stroma in the nasopharynx. Tumors with a similar histomorphology have been reported in extrapharyngeal sites, such as the stomach, salivary glands, thymus, biliary tract, colon, and oropharynx, where it has been referred to as lymphoepithelioma-like carcinoma (LELC). ${ }^{2,3}$ Only four cases of LELC have been reported in the gall bladder. ${ }^{2-5}$ This paper report an unusual case of Epstein-Barr virus (EBV) associated mixed gall bladder carcinoma exhibiting a distinct phenotype of LELC and ad- enocarcinoma with mucinous differentiation, which has not been reported previously. The index case underscores the importance of recognizing this histologic variant of gall bladder carcinoma as its response to surgical resection and chemotherapy in terms of the overall patient survival, and the prognosis might be different from a conventional adenocarcinoma.

\section{CASE REPORT}

A 45-year-old Indian woman presented to the department of Gastrointestinal Surgery with a complaint of abdominal pain in the upper abdomen for 2 months. It was not asso-

Received March 15, 2020. Revised April 30, 2020. Accepted May 5, 2020.

(C) This is an open access article distributed under the terms of the Creative Commons Attribution Non-Commercial License (http://creativecommons.org/licenses/ by-nc/4.0) which permits unrestricted non-commercial use, distribution, and reproduction in any medium, provided the original work is properly cited. Copyright (C) 2021. Korean Society of Gastroenterology.

Correspondence to: Surbhi Goyal, Department of Pathology, Govind Ballabh Pant Institute of Postgraduate Medical Education and Research, 1 Jawaharlal Nehru Marg, 64 Khamba, Raj Ghat, New Delhi, Delhi 110002, India. Tel: +91-9873896416, E-mail: dr.surbhi4you@gmail.com, ORCID: https://orcid.org/0000-0002-0554-9540 
ciated with jaundice, vomiting, or loss of weight/appetite. She had a prior history of Bell's palsy 2 years earlier and had recovered with some residual weakness. The clinical examination showed no significant findings. The contrast-enhanced CT scan revealed a heterogeneously enhancing mass measuring $4.8 \times 3.5 \mathrm{~cm}$ in the posterior wall of the neck and body of the gall bladder with liver infiltration in segment $\mathrm{V}$ (Fig. $1 \mathrm{~A}-\mathrm{C})$. No significant lymphadenopathy was noted. The tumor markers, CEA and CA-19.9, were markedly elevated with values $188.9 \mathrm{ng} / \mathrm{mL}(0-3 \mathrm{ng} / \mathrm{mL})$ and $109.3 \mathrm{IU} / \mathrm{L}$ (0-37 IU/L), respectively. The 18-F-fluorodeoxyglucose PET scan revealed similar findings with a very high standard uptake value (SUV) of 52.1 with no evidence of distant metastasis. A provisional diagnosis of a gall bladder carcinoma infiltrating the liver was made, and staging laparoscopy followed by radical cholecystectomy with a non-anatomical liver resection and standard lymphadenectomy was performed. The postoperative course was uneventful, and the patient has been started on cisplatin and gemcitabine.

A gross examination revealed a polypoidal growth, measuring
$4.5 \times 4 \times 3 \mathrm{~cm}$, arising from the posterior wall of the neck and body of the gall bladder, completely obliterating the lumen. The tumor was infiltrating into the adjacent liver parenchyma in the form of a well-circumscribed, homogenous gray-white solitary nodule measuring $3 \times 3 \times 2 \mathrm{~cm}$. On the cut, a few mucinous areas were identified (Fig. 1D, E). The cystic duct margin and liver resection margin were negative for a malignancy on frozen section analysis. Histopathology of the resected specimen revealed a malignant mixed tumor in the gall bladder comprising two phenotypically distinct components (Fig. 2A, $B)$. The predominant intraluminal polypoidal tumor was comprised of cells arranged in the anastomosing papillae and back-to-back glands (Fig. $2 \mathrm{C}$ ). The individual tumor cells were columnar to cuboidal with marked nuclear stratification, hyperchromasia, and conspicuous nucleoli (Fig. 2C). The mitotic count was 10-15/high power field. Extracellular mucin was conspicuous, but it accounted for less than $50 \%$ of the entire tumor, suggesting an adenocarcinoma with mucinous differentiation (Fig. 2D). The adjacent lining epithelium in the body of the gall bladder revealed high-grade dysplasia and intestinal
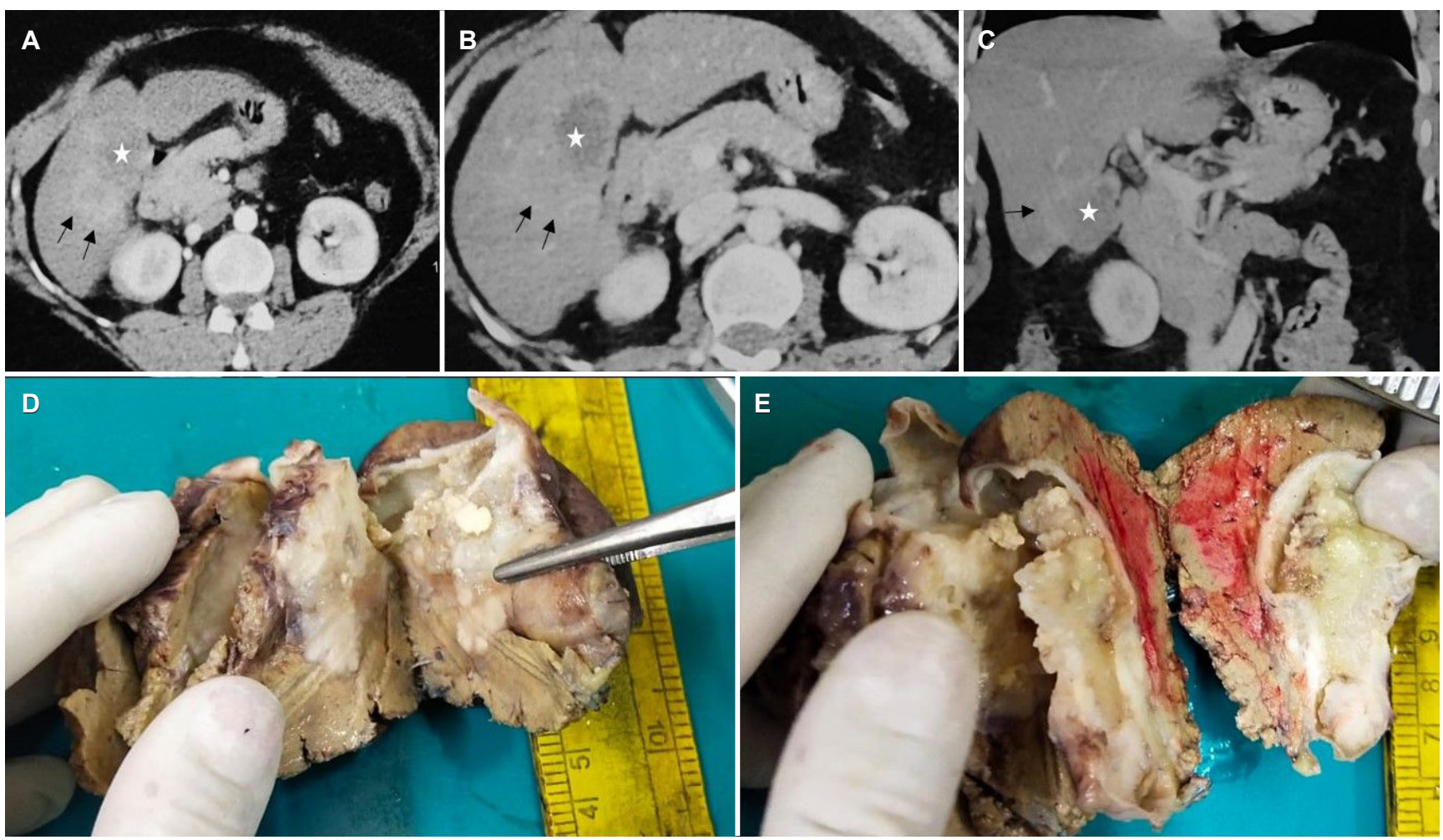

Fig. 1. (A) Axial arterial and (B) venous phase and (C) coronal venous phase contrast-enhanced CT images show heterogeneously and intensely enhancing mass involving the body of gall bladder (asterisk) with contiguous infiltration into segment 5 of the liver (arrows). Gross photograph shows $(D)$ a gray white growth in the posterior wall of the gall bladder infiltrating the adjacent liver parenchyma. (E) On cut, gall bladder lumen showing a polypoidal growth measuring $4.5 \times 4 \times 3 \mathrm{~cm}$ with mucinous areas. 

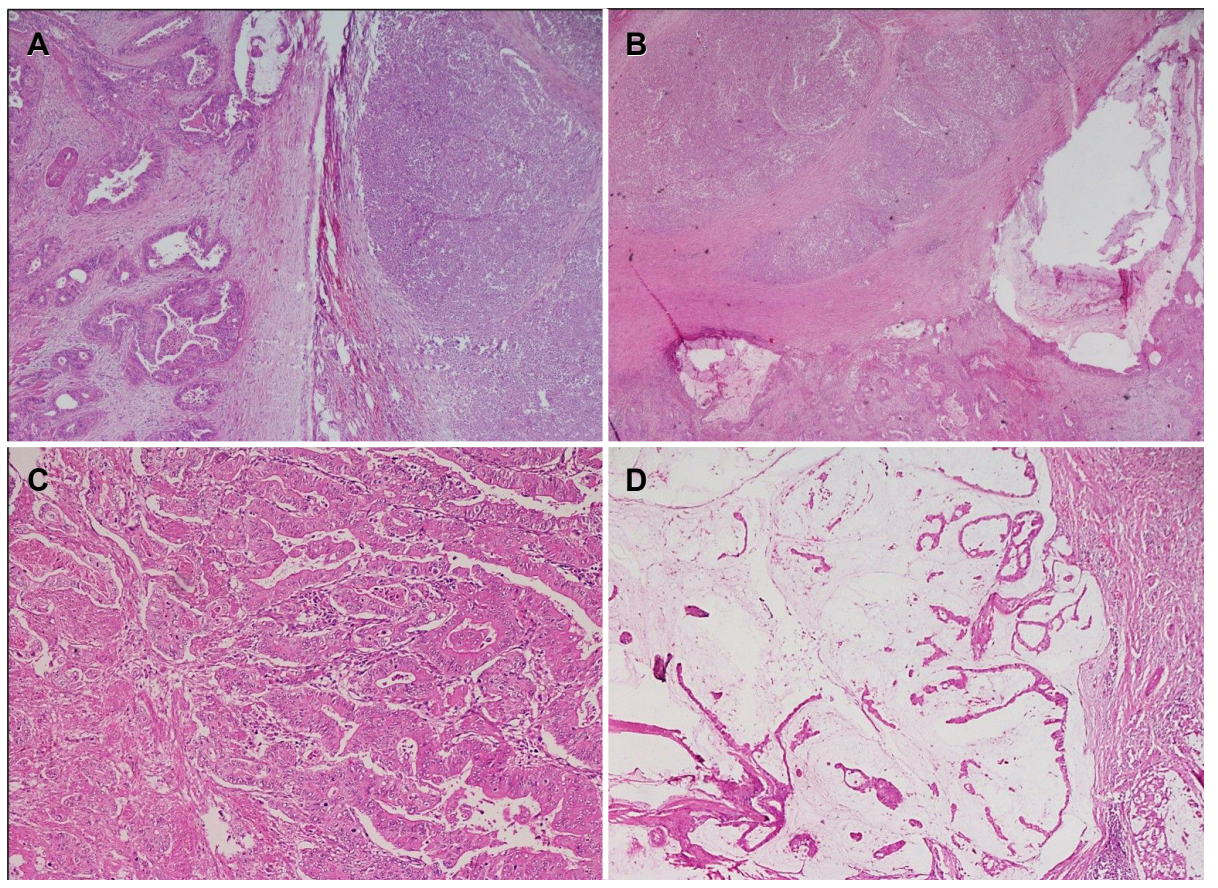

Fig. 2. (A) Section shows two phenotypically distinct components with LELC on the right side and an adenocarcinoma on the left (H\&E, $\times 40)$. (B) LELC on the left side with adenocarcinoma showing glands and mucinous areas in the right half (H\&E, $\times 40)$. (C) Higher magnification shows tumor arranged in anastomosing papillae and back-to-back arranged glandular pattern (H\&E, $\times 200$ ). (D) Areas of adenocarcinoma comprised of conspicuous extracellular mucin pools with floating tumor cells within them (H\&E, $\times 40)$.

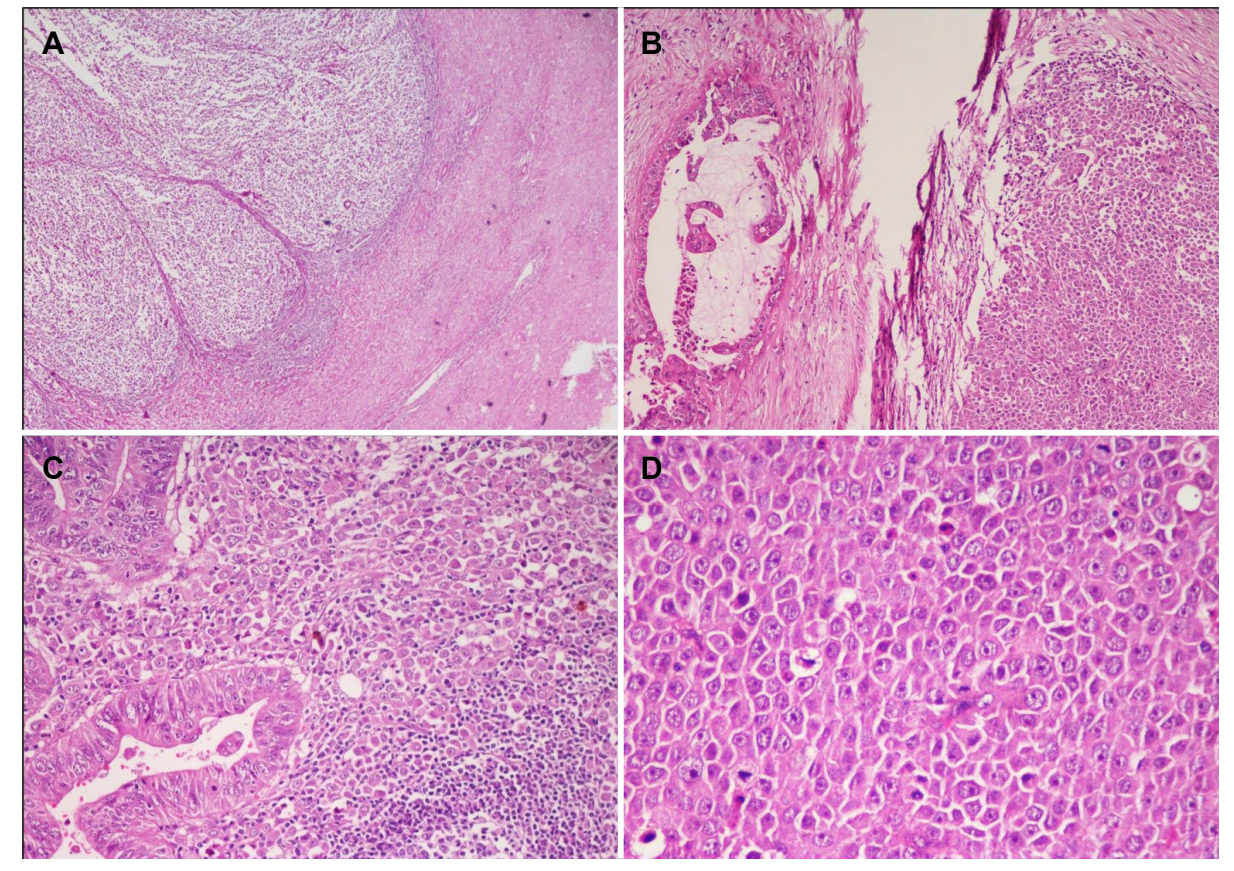

Fig. 3. Microphotographs show (A) an expansile, well-circumscribed tumor arranged in diffuse sheets and infiltrating the liver with broad pushing borders and dense peritumoral lymphoid infiltrate $(H \& E, \times 40)$. (B) Higher magnification showing the two distinct components comprised a dilated mucin-filled tumor gland on the left with lymphoepithelioma-like carcinoma (LELC) on the right side (H\&E, $\times 100)$. (C) LELC component comprised of cells arranged in syncytial sheets having prominent intratumoral and peritumoral lymphocytic infiltrate with the formation of many lymphoid follicles. Few dysplastic glands lined by columnar to cuboidal cells with marked nuclear stratification, hyperchromasia, and conspicuous nucleoli are seen at the junction of two components (H\&E, $\times 200)$. (D) Higher magnification of LELC areas shows tumor cells present in the syncytial sheets. These have moderate eosinophilic cytoplasm indistinct cell boundaries, large vesicular round to oval-shaped nuclei with single large prominent nucleoli $(\mathrm{H} \& \mathrm{E}, \times 400)$. 
metaplasia. The other component was a distinct tumor present in the syncytial sheets deeper within the gall bladder wall and infiltrating into the liver (Fig. 3A, B). These cells had moderate eosinophilic cytoplasm, indistinct cell boundaries, large vesicular round to oval-shaped nuclei with single large prominent nucleoli (Fig. 3C, D). A prominent peritumoral lymphocytic infiltrate with the formation of many lymphoid follicles was noted (Fig. 3A). The mitotic count was 8-10/high power field in these areas. A lymphovascular invasion was identified, but no perineural invasion could be seen.
Sections from the gall bladder-liver interface revealed the tumor infiltrating the liver with broad pushing margins, arranged in syncytial sheets and brisk peritumoral lymphocytic infiltrate. Only one of the three hepatoduodenal lymph nodes revealed tumor metastasis. On immunohistochemistry, the tumor cells were positive for pancytokeratin and cytokeratin7 (Fig. 4B) and negative for cytokeratin20, synaptophysin, chromogranin, and leukocyte common antigen (LCA). An admixture of CD3 positive and CD20 positive lymphocytes was observed at the advancing edge, reflecting the polyclonal nature (Fig.

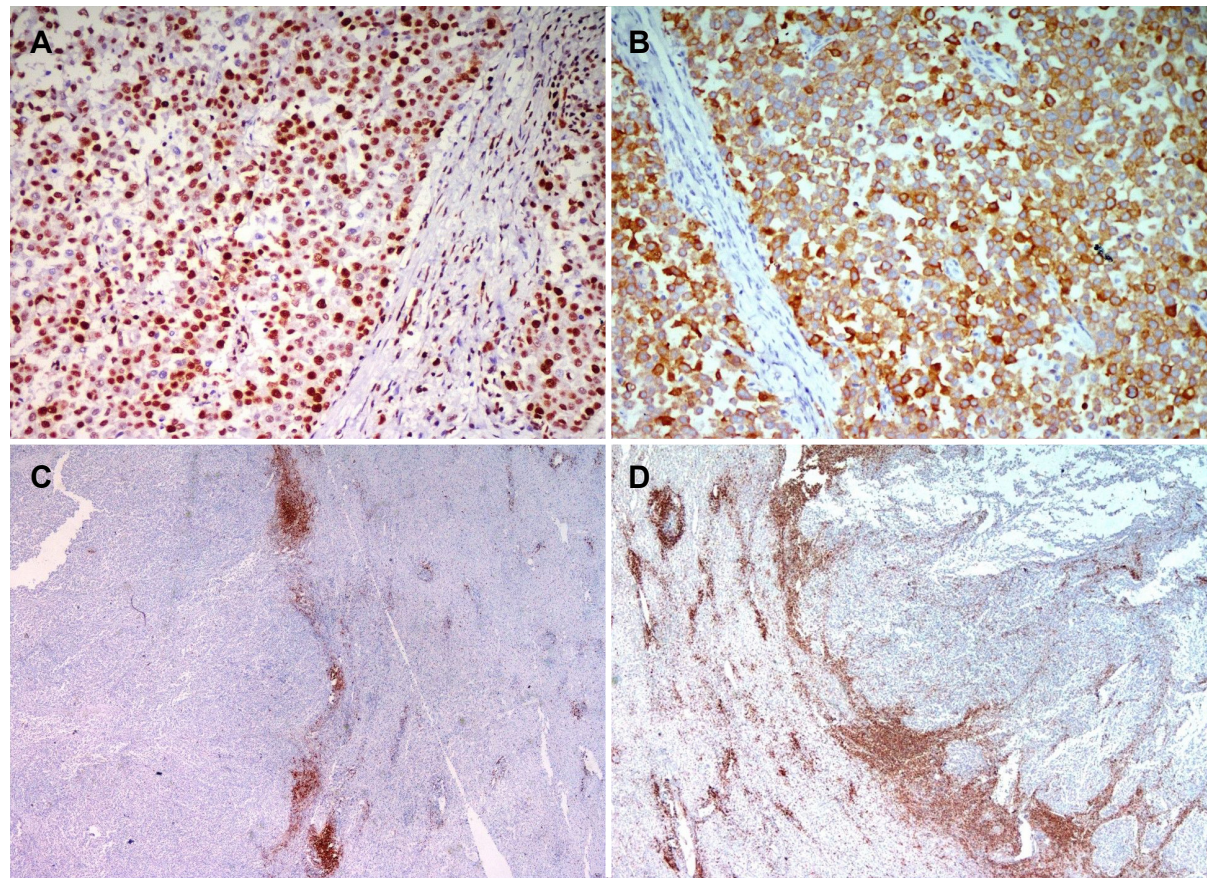

Fig. 4. (A) Tumor cells in the LELC areas show retained nuclear expression of MLH1. MSH2, MSH6, and PMS2 also showed similar findings in both the components (MLH1; IHC, $\times 100$ ). (B) LELC cells showing strong membranous staining with cytokeratin 7 (CK7; IHC, $\times 100)$. (C, D) Photomicrographs show a sprinkling of CD20 and CD3 positive lymphocytes in the peritumoral infiltrate $(C D 20 ; I H C, \times 20, C D 3$; IHC, $\times 20)$.

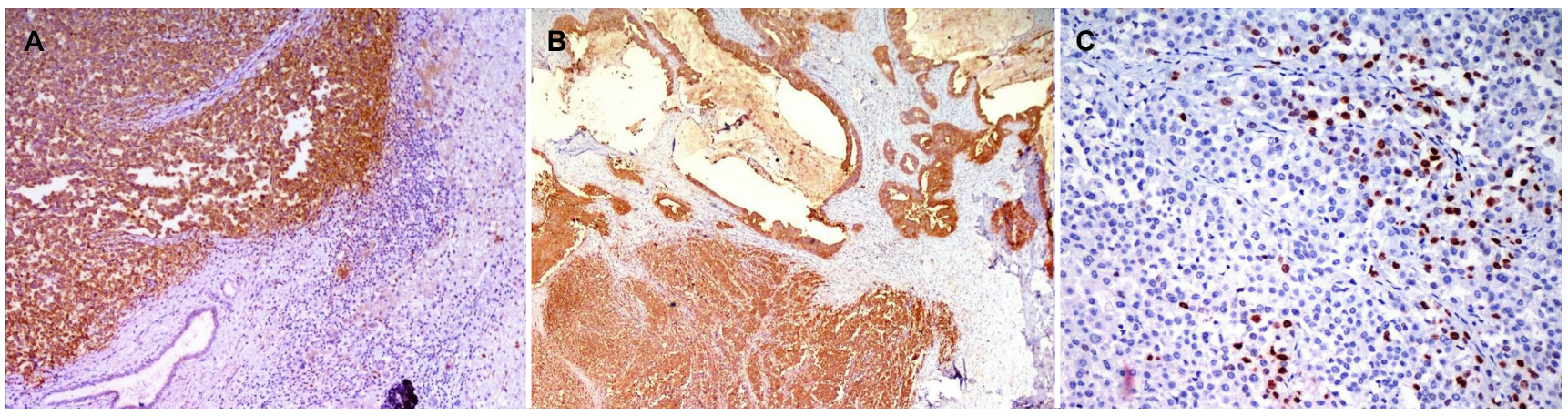

Fig. 5. (A) Strong granular membranous and cytoplasmic expression of latent membrane protein-1 (LMP-1) in the lymphoepithelioma-like carcinoma (LELC) component. Note the negative expression in the surrounding infiltrating lymphocytes and benign bile duct (LMP1; IHC, $\times 200)$. (B) Components of adenocarcinoma and LELC both showing strong cytoplasmic and membranous expression of latent membrane protein-1 (LMP1; IHC, $\times 40)$. (C) Positive nuclear expression of EBV encoded RNA on chromogenic in situ hybridization (EBER CISH, $\times 200)$. 
4C, D). The tumor cells showed the retained expression of MSH-2, MSH-6, MLH1, and PMS-2, indicating the microsatellite stable phenotype (Fig. 4A). The tumor cells showed strong granular membranous and cytoplasmic expression of latent membrane protein-1 (LMP-1) in both components, but the surrounding lymphocytes and non-neoplastic gall bladder mucosa were negative (Fig. 5A, B). Nuclear positivity for the EBV encoded RNA1 chromogen in-situ hybridization confirmed the EBV association (Fig. 5C).

Based on the morphology and immunohistochemistry, a final diagnosis of EBV associated mixed carcinoma-lymphoepithelioma-like carcinoma (LELC) and adenocarcinoma with mucinous differentiation, stage pT3N1MO was made. Extensive sampling was performed, and each component accounted for approximately $50 \%$ of the entire tumor.

\section{DISCUSSION}

In 1921, Schmincke ${ }^{1}$ first described lymphoepithelioma or undifferentiated carcinoma with lymphoid stroma in the nasopharynx characterized by two classical microscopic patterns Regaud and Schmincke pattern. In the Regaud pattern, the tumor cells form well-defined, cohesive cell nests and cords surrounded by dense lymphocytic inflammation, whereas in the latter, inflammatory cells permeate the cell nests to a greater extent. ${ }^{1}$ Typically, these tumors consist of malignant cells arranged in diffuse sheets and syncytial patterns with a dense lymphocytic inflammatory infiltrate at the tumor edge.
They typically lack desmoplastic stroma and have pushing borders. The tumor cells have moderate eosinophilic cytoplasm, indistinct cell borders, vesicular nuclei, and prominent nucleoli. ${ }^{6}$ Tumors with a similar morphology have also been reported at other extrapharyngeal sites, which have been referred to as lymphoepithelioma-like carcinoma or medullary carcinoma. $^{2,3,7}$ This case showed the typical morphological features of the Regaud pattern of lymphoepithelioma of the nasopharynx.

The reported incidence of LELC in various extrapharyngeal sites is variable, with the highest in the stomach (6-8\%) followed by the colon (4\%), breast (2-5\%), and biliary tract (3\%). LELC is rare in the urinary bladder $(0.4-1.3 \%)$, lungs $(<1 \%)$, esophagus $(<1 \%)$, salivary glands $(<1 \%)$, liver $(<1 \%)$, and pancreas $(<1 \%)$. $^{8-11}$ To the best of the authors' knowledge, only four cases of LELC in the gall bladder have been reported. $Y$ Muto et al. reported the first case in the gall bladder in 1984, and they have used the term carcinoma with lymphoid stroma. ${ }^{4}$ The recent most case was reported by Choi and $\mathrm{Lim}^{2}$ in 2016, which was the first case of mixed adenocarcinoma and LELC, but there was no EBV association, liver involvement, or lymph node metastasis, unlike our case (Table 1).

Association of EBV infection with lymphoepithelioma is well established in the nasopharynx and is seen in up to $80-100 \%$ of cases. $^{3,12}$ In contrast, at the extrapharyngeal sites, this association with EBV is very inconsistent. EBV is commonly detected in the stomach (80\%), lung (79\%), and salivary gland malignancies but rarely in the esophagus. ${ }^{2,3,7,8,11}$ By contrast,

Table 1. LELC of the Gall Bladder-Review of Literature

\begin{tabular}{|c|c|c|c|c|c|}
\hline Parameter & Muto et al. $(1984)^{4}$ & Todd et al. $(1996)^{3}$ & Sinha et al. $(2014)^{5}$ & Choi and $\operatorname{Lim}(2016)^{2}$ & Present case (2021) \\
\hline Age (years) & 76 & 60 & 64 & 83 & 45 \\
\hline Sex & Male & Female & Female & Male & Female \\
\hline Size $(\mathrm{cm})$ & Hen's egg & $10.5 \times 7.5 \times 6$ & 3 & $3 \times 4$ & $4.5 \times 4 \times 3 \mathrm{~cm}$ \\
\hline Site (Gall bladder) & Body \& Fundus & - & Neck & - & Neck \& Body \\
\hline Type & Pure LELC & Pure LELC & Pure LELC & $\begin{array}{c}\text { Mixed } \\
\text { LELC+Adenocarcinoma }\end{array}$ & $\begin{array}{c}\text { Mixed LELC+Mucinous } \\
\text { adenocarcinoma }\end{array}$ \\
\hline PNI & - & - & - & Present & Absent \\
\hline LVI & - & - & - & Absent & Present \\
\hline Liver involvement & Absent & - & Absent & Absent & Present \\
\hline Lymph node metastasis & Absent & - & Absent & Absent & Present \\
\hline EBV association & - & Absent & Absent & Absent & Present \\
\hline MSI & - & - & - & - & Retained \\
\hline
\end{tabular}

LELC, Iymphoepithelioma-like carcinoma; PNI, perineural invasion; LVI, lymphovascular invasion; EBV, Ebstein-Barr virus; MSI, microsatellite instability. 
there are no reports of the EBV in gall bladder, breast, hepatobiliary tract, skin, and urinary bladder carcinoma. ${ }^{2,3}$

Lee et al. $^{13}$, in a meta-analysis, found that the EBV was associated with $80 \%$ of LELC and $6 \%$ of non-LEL adenocarcinoma in the stomach. They also found that an EBV infection was significantly associated with a young age, male gender, diffuse histological type of gastric carcinoma, LELC, and a CpG island methylator phenotype-high status, but not associated with the clinical stage, lymph node metastasis, and $H$. pylori infection. ${ }^{13}$

EBV can be detected by the expression of a set of viral gene products, comprised of six EBV nuclear antigens (EBNAs 1, 2, 3A, 3B, 3C, and -LP), three latent membrane proteins (LMPs 1, 2A, and 2B), two EBV-encoded small non-coding RNAs (EBERs 1 and 2) and the BamHI A rightward transcripts (BARTs). Cheng et al. reported that EBV-positive gastric LELCs expressed EBNA1 rather than LMP1 and EBNA2, similar to Burkitt lymphoma indicating latency pattern type $1^{12,14,15}$ In the present case, however, LMP1 and EBER RNAs were found to be positive, indicating latency pattern type 2 and 3 , which is characteristic of a nasopharyngeal carcinoma and lymphoproliferative disease.

Microsatellite instability-high (MSI-H) has been found in $85 \%$ of colonic LELC. $^{9}$ There is no association between MSI and LELC in the breast, while this association is variable in the pancreas. Gastric LELC consists of two subsets, EpsteinBarr virus (EBV)-positive and MSI-H carcinomas, both of which are mutually exclusive. ${ }^{8,13}$ Grogg et al. ${ }^{16}$, in a study of 117 gastric cancer patients, found that EBV was absent in all MSI-H cases, and only $7.5 \%$ of microsatellite-stable cases harbored EBV. On the other hand, in gall bladder LELC, the association with MSI deficiency has not been documented.

LELC usually has a more favorable prognosis than conventional adenocarcinoma or squamous cell carcinoma, irrespective of the site..$^{2,7,8,11,17-19}$ Furthermore, in a meta-analysis, EB- positive gastric LEL had better overall survival and prognosis than EBV-negative LEL, particularly in advanced stages. The possible reasons might be the increased sensitivity of EBV-positive tumor cells to chemotherapy-induced apoptosis, as observed in EBV-associated Hodgkin lymphoma. Song et al. $^{20}$ reported the 12-year disease-free survival in gastric LELC to be approximately $95 \%$.

The standard treatment protocols for LELC have not been established, with surgical resection being the mainstay of treatment. In advanced stages, adjuvant chemotherapy (gemcitabine-cisplatin/carboplatin) is considered as a promising option. The remarkable tumor sensitivity to chemotherapy and radiotherapy in nasopharyngeal lymphoepithelioma might be extrapolated to LELC of extrapharyngeal sites and offers survival benefit in such patients compared to conventional carcinoma. ${ }^{3,11,12,19}$

To the best of the authors' knowledge, this is the first case of dual phenotype comprised of LELC and mucin secreting adenocarcinoma of the gall bladder, which has shown EBV association. The similar latency pattern of EBV expression to nasopharyngeal lymphoepithelioma in the present case might postulate a similar underlying etiopathogenesis, tumor biology, and the response to chemotherapy. Despite the exceptional infiltration of liver and nodal metastases by LELC, the intense EBV-associated lymphocytic response might limit the disease spread and confer better overall survival and prognosis in such cases. On the other hand, long-term follow-up studies will be needed to understand the biologic behavior and evaluate the possible therapeutic options and prognostic factors in EBV-associated LELC.

\section{REFERENCES}

1. Schmincke A. Über lymphoepitheliale Geschwülste. Beitr pathol Anat Allg Pathol 1921;68:161-170.

2. Choi NK, Lim SC. Mixed lymphoepithelioma-like carcinoma and adenocarcinoma of the gallbladder. Korean J Hepatobiliary Pancreat Surg 2016;20:148-151.

3. Todd DL, Ro JY, Gulley ML, Ayala AG. Lymphoepitheliomalike carcinoma of the gallbladder. Int J Surg Pathol 1996;4:183-187.

4. Muto Y, Sho Y, Uchimura M. Carcinoma with lymphoid stroma of the gallbladder--a case report. Jpn J Surg 1984;14:399-404.

5. Sinha PK, Mangla V, Behari C, Rastogi A, Chattopdhyay TK. Lymphoepithelioma-like carcinoma: an unusual gall bladder tumour. Trop Gastroenterol 2014;35:182-183.

6. Carbone A, Micheau C. Pitfalls in microscopic diagnosis of undifferentiated carcinoma of nasopharyngeal type (lymphoepithelioma). Cancer 1982;50:1344-1351.

7. Lauwers GY. Epithelial Neoplasms of the Stomach. In: Odze RD, Goldblum JR, eds. Odze and Goldblum surgical pathology of the Gl tract, liver, biliary tract and pancreas. 3rd ed. Philadephia: Elsevier Health Sciences, 2015:714.

8. Herath $\mathrm{CH}$, Chetty R. Epstein-Barr virus-associated lymphoepithelioma-like gastric carcinoma. Arch Pathol Lab Med 2008;132: 706-709.

9. Zenger S, Gurbuz B, Can U, et al. Clinicopathological importance of colorectal medullary carcinoma. Eur Surg 2019;51:308-314.

10. Lakhani SR, Ellis IO, Schnitt SJ, Tan PH, van de Vijver MJ. WHO classification of tumours of the breast. 4th ed. Lyon: IARC Press, 
2012.

11. Chen B, Chen X, Zhou P, et al. Primary pulmonary lymphoepithelioma-like carcinoma: a rare type of lung cancer with a favorable outcome in comparison to squamous carcinoma. Respir Res 2019;20:262.

12. Chou J, Lin YC, Kim J, et al. Nasopharyngeal carcinoma--review of the molecular mechanisms of tumorigenesis. Head Neck 2008;30:946-963.

13. Lee JH, Kim SH, Han SH, An JS, Lee ES, Kim YS. Clinicopathological and molecular characteristics of Epstein-Barr virus-associated gastric carcinoma: a meta-analysis. J Gastroenterol Hepatol 2009;24:354-365.

14. Cheng N, Hui DY, Liu Y, et al. Is gastric lymphoepithelioma-like carcinoma a special subtype of EBV-associated gastric carcinoma? New insight based on clinicopathological features and EBV genome polymorphisms. Gastric Cancer 2015;18:246-255.

15. Tsao SW, Tramoutanis G, Dawson CW, Lo AK, Huang DP. The significance of LMP1 expression in nasopharyngeal carcinoma. Semin Cancer Biol 2002;12:473-487.
16. Grogg KL, Lohse CM, Pankratz VS, Halling KC, Smyrk TC. Lymphocyte-rich gastric cancer: associations with Epstein-Barr virus, microsatellite instability, histology, and survival. Mod Pathol 2003;16:641-651.

17. Thirunavukarasu P, Sathaiah M, Singla S, et al. Medullary carcinoma of the large intestine: a population based analysis. Int J Oncol 2010;37:901-907.

18. Tamas EF, Nielsen ME, Schoenberg MP, Epstein Jl. Lymphoepithelioma-like carcinoma of the urinary tract: a clinicopathological study of 30 pure and mixed cases. Mod Pathol 2007;20:828-834.

19. Qian XH, Zhou DK, Wang WL. Surgical treatment of Epstein-Barr virus-associated lymphoepithelioma-like carcinoma occurring in both the posterior mediastinum and liver: case report. Medicine (Baltimore) 2020;99:e23610.

20. Song HJ, Srivastava A, Lee J, et al. Host inflammatory response predicts survival of patients with Epstein-Barr virus-associated gastric carcinoma. Gastroenterology 2010;139:84-92.e2. 